\title{
"Study of Treadmill test in Detecting Asymptomatic Coronary Artery Disease in Type 2 Diabetes Mellitus"
}

\author{
Dr.K.Swaminathan ${ }^{1}$, Dr.M.Gayathri ${ }^{2}$ \\ ${ }^{1}$ Professor, Department of General Medicine, Coimbatore Medical College \& Hospital, Coimbatore. \\ ${ }^{2}$ Senior Resident, Department of General Medicine, Coimbatore Medical College \& Hospital Coimbatore.
}

\begin{abstract}
Coronary arteryDisease is the common impact of death worldwide in type 2 Diabetes mellitus.High rate of asymptomatic coronary heart disease in type $2 \mathrm{DM}$. We studied aboutthe prevalence of silent myocardial ischemia (SMI) in asymptomatic type 2 diabetes mellitus by using treadmill test. We also analysed about the clinical predictions ofSilent Myocardial Ischemia in asymptomatic type 2 DM. The study was one year cross sectional study conducted on the 50 asymptomatic type 2 DM outpatients in the tertiary care hospital during the period of July 2014 to July 2015. The result showed that Out of 50 cases, Treadmill test positive cases were $15(30 \%)$ and negative cases were 35(70\%) patients groups.The preponderance of SMI in type 2DM, without previous clinical and electrocardiographic signs of ischemic heart disease or hypertension is 30\%.Age, duration of diabetes, triglyceride levels, autonomic neuropathy and glycosylated hemoglobin are strong clinical predictors of silent myocardial ischemia.Based on our study, a routinescreening is must for Silent Myocardial ischemia in asymptomatic type 2 DM outpatients who had longer duration of diabetes, high triglycerides and HbAlC levels.
\end{abstract}

Keywords: Coronary Artery Disease, Silent Myocardial ischemia, Type 2 Diabetes Mellitus, Tread Mill Test.

\section{Introduction}

Diabetes Mellitus (DM) is a disordered metabolic syndrome with hyperglycaemia either due to defect in insulin secretion or insulin resistance. It may cause progressive tissue damage and both micro and macrovascular complications. More than $95 \%$ of diabetes are type 2 DM. They always had insidious, latent, along with asymptomatic phase. Therefore management of DM has changed not only controlling symptoms but also preventing complications.

BMI (Body Mass Index) of any indian of more than 23 must be investigated for diabetes ${ }^{1}$. The waist circumference of more than $85 \mathrm{~cm}$ in males and $80 \mathrm{~cm}$ in females are the persons must be screened for DM. The yearly screening for glycated hemoglobin and TOD (Target organ damage) is a must. Coronary heart disease, atherosclerotic CHD, is the common impact of death worldwide in type 2 DM.INTERHEART $-10 \%$ of the population attributable risk of a first MI. High rate of asymptomatic coronary disease in type 2 DM. There will be a reduced myocardial flow reserve in type 2 DM. Hyperglycaemia decreases endothelium-derived NO availability and affects vascular function mainly through the increased production of ROS. Asymptomatic Diabetes mellitus higher median CAC scores raising frequency of silent ST segment depression and coronary perfusion changes during exercise testing. Stress treadmill test is a readily available, cost effective, first line test for identification of coronary heart disease in DM of longer duration without any symptoms of angina. This study was outlined to determine SMI (Silent Myocardial Ischemia) in selected asymptomatic DM outpatients by exercise tread mill test. ${ }^{2}$

1. To study the prevalence of silent myocardial ischemia in asymptomatic type 2 DM patients by using tread mill test.

2 .To analyse the clinical predictions of silentmyocardial ischemia in asymptomatic type $2 \mathrm{DM}$ patients.

\section{Materials And Methods}

The study was conducted in the Tertiary care Hospital, during the period of July2014 to July 2015. 1.1 Study Design:

The study was one year cross sectional study on the asymptomatic type 2 DM outpatients beyond clinical and ECG evidence of CAD.

\subsection{Source Of Data:}

The 50 outpatients of asymptomatic type $2 \mathrm{DM}$ beyond clinical evidence of CAD attending, diabetology and medicine OPD at Tertiary Care Hospital, were enrolled in the study. 


\subsection{Sample Size:}

A samplesize of 50 patients were included in the study.

\subsection{Selection Criteria:}

1.All type 2diabetic outpatients (both sex ) in the age group of 35-60 yrsof age .

2.Asymptomatic outpatients with type 2 DM with normal resting ECG.

\subsection{Exclusion CRITERIA:}

1. Previous history of MI, heart failure.

2. Evidence of angina pectoris

3. Anaemia

4. Hypertension

5. Renal disease

6. ECG evidence of Q wave MI, ischemic ST-segment or T wave abnormality or complete LBBB.

7. Any chronic illness because of cancer and ESRD or liver disease.

\section{Results}

We studied around 50 outpatients of asymptomatic type 2 DM beyond clinical and electrocardiographic documentation of CAD in Coimbatore Medical college hospital, Coimbatore, and following results were noted.

Table -1: Durationof Diabetes Mellitus:

\begin{tabular}{|l|l|l|}
\hline Duration of DM & N \\
\hline Duration & 26 & $(\%)$ \\
\hline$<5$ years & 12 & $52 \%$ \\
\hline $6-10$ & 7 & $24 \%$ \\
\hline $11-15$ & 5 & $14 \%$ \\
\hline$>15$ years & 50 & $10 \%$ \\
\hline Total & $100 \%$ \\
\hline
\end{tabular}

This table shows, more number of patients (26 i.e., 52\%) were having diabetes equal to or less than 5 years, followed by 12 patients (24\%) with the duration of 6-10 years, next 7 patients (14\%) between 11-15 years and only 5 patients (10\%) between 16-20 years. In the study population, more number of patients (26 i.e., 52\%) were having diabetes equal to or less than 5 years, followed by 12 patients (24\%) with the duration of 6-10 years, next 7 patients (14\%) between 11-15 years and only 5 patients $(10 \%)$ between $16-20$ years.

Table- 2: Glycosylatedhaemoglobin and duration of diabetes mellitus.

\begin{tabular}{|c|c|c|c|c|c|c|}
\hline \multicolumn{7}{|c|}{ Association of HbA1c with Duration of DM [N=50] } \\
\hline \multirow[b]{2}{*}{ HbA1c } & \multicolumn{4}{|c|}{ Duration of DM } & \multirow[b]{2}{*}{ Total } & \multirow[b]{2}{*}{$(\%)$} \\
\hline & $<5$ years & $6-10$ & $11-15$ & $>15$ years & & \\
\hline $6-8$ & 21 & 1 & 2 & 0 & 24 & $48 \%$ \\
\hline $8-10$ & 4 & 10 & 2 & 0 & 16 & $32 \%$ \\
\hline $10-12$ & 1 & 1 & 3 & 5 & 10 & $20 \%$ \\
\hline Total & 26 & 12 & 7 & 5 & 50 & \\
\hline
\end{tabular}

This table shows as there is increase in duration of DM ,there is increase in $\mathrm{HbA1C}$ level. Out of 5 patients of more than 15 yrs duration of DM those glycemic index are very high around 10-12. It shows 5/5 (100\%) ofpatients having poor glycemic index. This shows as the duration of DM increased, there is a increase in $\mathrm{HbA1C}$ level .This shows poor glycaemic index. There is a statistically significant difference between duration of diabetes and $\mathrm{HbA1C}$ level

Table - 3: TMT results.

\begin{tabular}{|c|c|c|}
\hline \multicolumn{3}{|c|}{ TMT RESULTS } \\
\hline TMT & $\mathrm{N}$ & $(\%)$ \\
\hline POSITIVE & 15 & $30 \%$ \\
\hline NEGATIVE & 35 & $70 \%$ \\
\hline Total & 50 & $100 \%$ \\
\hline
\end{tabular}

Out of 50 patients, Treadmill test was positive in $15(30 \%)$ and Treadmill test was negative in $35(70 \%)$ patients. 
Table - 4: TMT results and duration of diabetes mellitus.

\begin{tabular}{|l|l|l|l|l|}
\hline DURATION & POSITIVE & NEGATIVE & TOTAL & $(\%)$ \\
\hline$<5$ years & 3 & 22 & 26 & $52 \%$ \\
\hline $6-10$ & 3 & 9 & 12 & $24 \%$ \\
\hline $11-15$ & 5 & 2 & 7 & $14 \%$ \\
\hline$>15$ years & 4 & 1 & 5 & $10 \%$ \\
\hline TOTAL & 15 & 35 & 50 & \\
\hline
\end{tabular}

Out of 50 cases Treadmill test was positive in 15 (30\%) and negative in 35(70\%) patients. Tread mill test was positive in 4/26 (27\%), 3/9 (20\%), 5/7 (33\%) and 3/5(20\%) patient with duration of diabetes $\leq 5,6$ to 10,1 to 15 , and $16-20$ years respectively.

This shows the relation of silent myocardial ischemia and duration of diabetes mellitus. In the present study out of 50 cases Treadmill test was positive in $15(30 \%)$ and negative in $35(70 \%)$ patients. Tread mill test was positive in 4/26 (27\%), 3/9 (20\%), 5/7 (33\%) and 3/5(20\%) patient with duration of diabetes $\leq 5,6$ to 10,1 to 15 , and 16-20 years respectively. This shows longer the duration of diabetes, greater the risk of silent myocardial ischemia.

Table -5:Comparison of diabetic subjects with and without silent myocardial ischemia.

\begin{tabular}{|c|c|c|c|c|c|c|c|c|}
\hline \multicolumn{8}{|c|}{ Mean of Clinical Variables with TMT Results } & \multirow{3}{*}{$\mathrm{p}$ value } \\
\hline & & \multirow{2}{*}{ Mean } & \multirow{2}{*}{ SD } & \multicolumn{2}{|c|}{$95 \%$ CI for Mean } & \multirow{2}{*}{ Minimum } & \multirow{2}{*}{ Maximum } & \\
\hline Variables & TMT & & & Lower & Upper & & & \\
\hline \multirow{3}{*}{ Age } & Positive & 52.3 & 5.6 & 49.2 & 55.4 & 42 & 60 & \\
\hline & Negative & 47.1 & 6.2 & 44.9 & 49.2 & 35 & 60 & $<0.01$ \\
\hline & Total & 48.6 & 6.4 & 46.8 & 50.4 & 35 & 60 & \\
\hline \multirow{3}{*}{ Duration } & Positive & 10.4 & 5.3 & 7.5 & 13.3 & 2 & 18 & \\
\hline & Negative & 5.4 & 4.2 & 4.0 & 6.9 & 1 & 18 & $<0.01$ \\
\hline & Total & 6.9 & 5.1 & 5.5 & 8.4 & 1 & 18 & \\
\hline \multirow{3}{*}{ BMI } & Positive & 23.4 & 3.8 & 21.3 & 25.5 & 19.8 & 33.6 & \\
\hline & Negative & 25.9 & 3.9 & 24.6 & 27.3 & 19.4 & 32.3 & $<0.05$ \\
\hline & Total & 25.2 & 4.0 & 24.0 & 26.3 & 19.4 & 33.6 & \\
\hline \multirow{3}{*}{ Pulse } & Positive & 75.9 & 6.1 & 72.6 & 79.3 & 68 & 86 & \\
\hline & Negative & 76.0 & 6.0 & 73.9 & 78.1 & 68 & 90 & $>0.05$ \\
\hline & Total & 76.0 & 6.0 & 74.3 & 77.7 & 68 & 90 & \\
\hline \multirow{3}{*}{ SBP } & Positive & 127.9 & 3.2 & 126.1 & 129.6 & 120 & 130 & \\
\hline & Negative & 128.4 & 4.7 & 126.8 & 130.0 & 120 & 136 & $>0.05$ \\
\hline & Total & 128.2 & 4.3 & 127.0 & 129.5 & 120 & 136 & \\
\hline \multirow{3}{*}{ DBP } & Positive & 74.3 & 4.8 & 71.6 & 76.9 & 70 & 80 & \\
\hline & Negative & 75.3 & 5.4 & 73.5 & 77.2 & 60 & 84 & $>0.05$ \\
\hline & Total & 75.0 & 5.2 & 73.5 & 76.5 & 60 & 84 & \\
\hline \multirow{3}{*}{ FBS } & Positive & 170.5 & 33.1 & 152.2 & 188.9 & 126 & 256 & \\
\hline & Negative & 183.0 & 40.3 & 169.1 & 196.8 & 125 & 291 & $>0.05$ \\
\hline & Total & 179.2 & 38.4 & 168.3 & 190.2 & 125 & 291 & \\
\hline \multirow{3}{*}{ PPBS } & Positive & 224.6 & 53.6 & 194.9 & 254.3 & 170 & 325 & \\
\hline & Negative & 227.1 & 75.5 & 201.1 & 253.0 & 30 & 385 & $>0.05$ \\
\hline & Total & 226.3 & 69.1 & 206.7 & 246.0 & 30 & 385 & \\
\hline \multirow{3}{*}{ Cholesterol } & Positive & 174.4 & 31.8 & 156.8 & 192.0 & 133 & 234 & \\
\hline & Negative & 180.9 & 28.5 & 171.2 & 190.7 & 133 & 234 & $>0.05$ \\
\hline & Total & 179.0 & 29.3 & 170.7 & 187.3 & 133 & 234 & \\
\hline \multirow{3}{*}{ TG } & Positive & 176.6 & 43.8 & 152.3 & 200.9 & 80 & 230 & \\
\hline & Negative & 130.5 & 30.9 & 119.8 & 141.1 & 80 & 190 & $<0.001$ \\
\hline & Total & 144.3 & 40.8 & 132.7 & 155.9 & 80 & 230 & \\
\hline \multirow{3}{*}{ Urea } & Positive & 27.3 & 3.5 & 25.4 & 29.2 & 22 & 36 & \\
\hline & Negative & 29.5 & 5.7 & 27.5 & 31.4 & 18 & 40 & $>0.05$ \\
\hline & Total & 28.8 & 5.2 & 27.3 & 30.3 & 18 & 40 & \\
\hline \multirow{3}{*}{ Creatinine } & Positive & 0.8 & 0.2 & 0.7 & 0.9 & 0.5 & 1.2 & \\
\hline & Negative & 0.8 & 0.2 & 0.8 & 0.9 & 0.5 & 1.3 & $>0.05$ \\
\hline & Total & 0.8 & 0.2 & 0.8 & 0.9 & 0.5 & 1.3 & \\
\hline \multirow{3}{*}{ HbA1c } & Positive & 9.7 & 1.3 & 9.0 & 10.4 & 7.2 & 11.6 & \\
\hline & Negative & 7.7 & 1.4 & 7.2 & 8.2 & 6.1 & 11.2 & $<0.001$ \\
\hline & Total & 8.3 & 1.6 & 7.8 & 8.8 & 6.1 & 11.6 & \\
\hline
\end{tabular}

The observation made with treadmill testing is as follows with reference to average duration of diabetes, HbA1C levels and triglycerides levels; It was found that there was a statistically significant difference in Tread mill test positive cases and Tread mill test negative cases with reference to the average age, duration of DM $(p=0.001)$, average HbA1C levels $(p=0.001)$ and triglycerides levels $(p=0.001)$.

Average duration of DM in Treadmill test positive and negative cases was 10.4and 5.4 years respectively. Average age of diabetic patients in Treadmill test positive and negative cases are 52.3 and 47.1 
respectively. Average HbA1C levels in Treadmill test positive and Treadmill test negative cases was 9.7\% and $7.7 \%$ respectively. Average triglycerides levels in Treadmill test positive and negative cases was 176.6 and $130.5 \mathrm{mg} \%$ respectively.

Table -6: Diabetic autonomic neuropathy and silent myocardial ischemia.

\begin{tabular}{|c|c|c|l|}
\hline Signs & \multicolumn{2}{|c|}{ TMT } & \\
\hline OH/ VM / SHG & POSITIVE & NEGATIVE & TOTAL \\
\hline Yes & 8 & 5 & 13 \\
\hline No & 7 & 30 & 37 \\
\hline TOTAL & 15 & 35 & 50 \\
\hline
\end{tabular}

$8(61.5 \%)$ outof $13 \mathrm{DM}$ with ANhad silent myocardial ischemia, while 7(18.9\%) out of 37 diabetes without AN had SMI. This shows DM with Autonomic neuropathyhad high occurrence of SMI than without diabetes. There is a significant difference between autonomic ne uropathy.

\section{Discussion}

Coronary Artery Disease detection in asymptomatic type $2 \mathrm{DM}$ is often delayed. The preponderance of SMI in type $2 \mathrm{DM}$ is variable and ranges from 9 to75\%., ${ }^{3,4}$ My study was anticipated at the asymptomatic presentation of CAD in the form of (SMI) in asymptomatic DM patients. It consisted of two aspects; First the prevalence of SMI in asymptomatic patients with type2 DM. Secondly to assess clinical predictors of SMI in these patients.

This study consists of 50 asymptomatic type 2 diabetics without clinical and electrocardiographic evidence of CAD and were evaluated for the preponderance of SMI by using exercise treadmill testing.

Out of 50 cases 39 were males and 11 were females. In the age group of 31-40 yrs of age 5 patients are present. 3 were males, 2 were females .In the age group of 41-50 yrs of age 26 were present, 22 were males, 4 were females. In the 51-60yrs of age group 14 were males, 5 were females. Most patientsin the age group of 41-50 yrs of age.

$25(50 \%)$ patients were with normal body mass index, 16 patients $(32 \%)$ were overweight while only $9(18 \%)$ patients were obese. In the study population, more number of patients (26 i.e., 52\%) were having diabetes equal to or less than 5 years, followed by 12 patients (24\%) with the duration of 6-10 years, next 7 patients (14\%) between 11-15 years and only 5 patients (10\%) between $16-20$ years.

As the duration of diabetes increased there is a increase in HbA1C level in cases of duration of diabetes $>15$ yrs duration of diabetes. This shows poor glycemic index. There is a statistically significant difference between duration of $\mathrm{DM}$ and $\mathrm{HbA1C}$ level.

In our50 patients, $2(8 \%)$ was on diet control alone, $25(50 \%)$ were on one or other OHA's, $11(22 \%)$ were on one or other form of insulin while $12(24 \%)$ were receiving both OHA's and insulin. There is a significant difference between duration of diabetes and treatment regimen.

Out of 50 cases TMT positive cases are $15(30 \%)$ and negative cases are $35(70 \%)$ patients. The study shows the prevalence of SMI in type 2 asymptomatic DM was turned out to be $30 \%(15 / 50)$.

Our findings were similar to previous studies. One study ${ }^{6}$ found that $29 \%$ diabetes who were asymptomatic for CAD had SMI on 24 hour ambulatory monitoring exercise electrocardiogram.Another similar study ${ }^{7}$ had shown higher predominance of SMI in DM as compared to non diabetics. Another study ${ }^{8}$ in India identified that $38.3 \%$ of DM beyond prior CAD had SMI on treadmill test.Another study ${ }^{8}$ from India, reported $50 \%$ incidence of silent myocardial ischemia in diabetics on exercise electrocardiogram and $35 \%$ on ambulatory monitoring. Yet another group ${ }^{9}$ found $12.1 \%$ of diabetics free of CAD to have SMI on exercise electrocardiogram testing. Another study ${ }^{10}$, found that SMI was positive in $14(46.7 \%$ ) out of $30 \mathrm{DM}$ patients by treadmill test.One more study population ${ }^{11}$ identified that a total of $113 / 522$ patients (22\%) had SMI using stress testing in asymptomatic patients with type 2 diabetes mellitus.

One more study $^{12}$ found that $62 / 500$ patients $(12.4 \%)$ had SMI in outpatients with type DM by using exercise electrocardiogram. So in my study it shows that DM patients had a higher prevalence of silent myocardial ischemia.

Durationof Type 2 Diabetes Mellitus And Silent Myocardialischemia.

In 50outpatients, Treadmill test positive cases are 15 patients (30\%) and negative cases are 35 patients $(70 \%)$.

- 26 patients with diabetes duration equal to or less than 5 years, Tread mill test was positive in $3(11 \%)$.

- 12 patients with diabetes duration between 6-10years. Tread mill test waspositive in 3(25\%).

- 7 patients with DM duration between 11 and 15 years Treadmill test positive in 5(71.4\%). 
- 5 patients with DM duration between 16 and 20 years Treadmill test was found to be positive in $4(80 \%)$.

Our results are similar to one study ${ }^{13}$ that found that $70 \%$ subjects $(7 / 10)$ with DM of $<5$ years duration had associated silent myocardial ischemia while only $30 \%$ subjects (3/10) with DM of $>5$ years duration had associated SMI.Another study ${ }^{14}$ including 500 patients with type 2 diabetes mellitus with normal resting ECG found that, 62(12.4\%) patients had asymptomatic coronary artery disease on exercise treadmill testing. The abnormalities of exercise test were associated with longer duration of $\operatorname{diabetes}(\mathrm{p}<0.005)$.

\section{Silent Myocardial Ischemia And Autonomic Neuropathy:}

In our study, $60 \%$ of subject s (8/13) with autonomic neuropathy (AN) had silent myocardial ischemia while only $18.9 \%$ of subjects (7/37) without autonomic neuropathy had myocardial ischemia. Hence it depicts that,DM patients with AN hadmuch higheroccurrence of SMI than those without it $(60 \%$ Vs $18.9 \%)$ of studies.

One group depictsthat in a DM population of already known or doubtful CAD had SMI much more common with AN (92\%Vs39\%).

Another study in India showed that $38.3 \%$ had SMI with a greater prevalence in those with AN ( 59\%) than those without it $(20 \%)$. Another studyfrom India found that occurrence of SMI was higher in patients with AN 12/13(40\%) compared to those without 3/30(10\%) p<0.001.

\section{Dyslipidemia And Silent Myocardial Ischemia:}

In the present study, we found average total cholesterol in TMT positive and negative cases were $174.4 \mathrm{mg} \%$ and $180.9 \mathrm{mg} \%$ respectively. Average triglyceride in Treadmill test positive and negative cases $176.6 \mathrm{mg} \%$ and $130.5 \mathrm{mg} \%$ respectively. Statistically significant value of $\mathrm{p}<0.001$ was found in triglycerides levels between both the groups.

The same results were observed in previous study ${ }^{17}$ which found that dyslipidemia was common in type 2DM and the most common aberrancy was elevated serum triglyceride levels $(73.3 \%)$. The next aberrancy was decreased serum HDL levels $(66.7 \%)$.Another study from India ${ }^{18}$, found that CAD had strong complementation with elevated levels of triglycerides (0.82) and low HDL (-0.81).Yet another study ${ }^{17}$ found that triglyceride levels were elevated in 28 treadmill positive cases compared to 15 treadmill negative cases $(\mathrm{p}<0.01)$.

\section{Glycosylated Hemoglobin And Silent Myocardial Ischemia}

The increased levels of glycosylated hemoglobin indicated poor glycemic control and it has great influence on CAD. In our study we found average $\mathrm{HbA1C}(\%)$ in Treadmill test positive and negative cases were 9.7and7.7 respectively. Statistically significant value of $\mathrm{p}<0.001$ was found in $\mathrm{HbA1C}(\%)$ levels between both the groups. One study ${ }^{18}$ found that among those who had diabetes mellitus, silent myocardial ischemia was present 27 of 54patients (50\%) who had hemoglobin A1clevel>or=7.6\% and in 39 of $137(28 \%)$ with hemoglobin A1c level ( $\mathrm{p}<0.005)$.In one more ${ }^{21}$ study, it shows eloquent increasing trend of HbA1c levels over the augmenting number of coronary vessels involvement with CAD ( $\mathrm{p}<0.0001)$.

\section{Conclusion}

In our study population, the preponderance of SMI in type 2DM, without previous clinical and electrocardiographic signs of ischemic heart disease or hypertension is 30\%.Duration of diabetes mellitus is directly proportional to increased risk of silent ischemia in type 2 DM. Triglyceride was found to be in higher levels in persons who had silent ischemia. Patients who had greater prevalence of silent myocardial ischemia on TMT were found to have higher glycosylated hemoglobin levels.

Diabetics with clinical signs of autonomic neuropathy had higher incidence of silent myocardial ischemia. Age, duration of diabetes, triglyceride levels, autonomic neuropathy and glycosylated hemoglobin are strong clinical predictors of silent myocardial ischemia. Early detection of outpatients with type 2 DM for SMI may prevent catastrophic cardiac events.Based on our study, a routine screening for Silent Myocardial Ischemia in asymptomatic type $2 \mathrm{DM}$ outpatients who had longer duration of diabetes, high triglycerides and HbA1C levels.

\section{Bibiliography}

[1]. American Diabetes Association-Clinical Practice Recommendations Diabetescare2007;30(Suppl.4).

[2]. Fauci, Braunwald, Kasper, Hauser Longo,JamesonLoscalzo. Harrison's principles ofinternal medicine; Diabetes mellitus. $17^{\text {th }}$ edn.,NewDelhi: McGraw Hill Medical Publishing Division,2008.

[3]. Gupta SB, Pandit RB. Silent myocardial ischemia and cardiac autonomic neuropathy I diabetes. Ind Heart J 1993; 44(4): 227-9.

[4]. Ahluwalia G, Jain P, Chugh SK, Wasir HS, Kaul U. SMI in diabetics with normal autonomic function. Int J Cardiol 1995; 
48(2): 147-53.

[5]. Misad Group. Prevalence of unrecognized SMI and its association with atherosclerotic risk factors in non insulin dependent DM. Am J Cardiol 1997; 79(2): 134-9.

[6]. Sukhija R, Chanwal D, Gambhir DS, Dewan R. Silent myocardial ischaemia in patients with type II diabetes mellitus and its relation with autonomic dysfunction. Indian Heart J 2000; 52(5): 540-6.

[7]. Wackers FJ, Young LH, Inzucchi SE, Chyun Da, Davey JA. Barrett EJ et al. Detection of Ischemia in Asymptomatic Diabetes Investigators. Detection of silent myocardial ischemia in asymptomatic diabetic subjects : the DIAD study. Diabetes Care 2004, 27( 8): 1954-61.

[8]. Sargin H, Ozisik M, Oxisik NC, Seven O, Orbay E, Gozu H et al. The prevalence of silent ischemia in Turkish patients with type 2 diabetes mellitus. Tohoku J. Exp med. 2005; 20594: 351-5.

[9]. Ahuwalia V. Incidence of silent myocardial ischemic in non insulin dependent diabetes mellitus; 1998.

[10]. Murray DP, O'Brien T, Mulrooney R, O'Sullivan. Autonomic dysfunction and silent myocardial ischemia on exercise testing in diabetes mellitus. Diabetic Med. 1990;8(7):580

[11]. Langer A, Freeman MR, Josse RG, Steiner G, Armstrong PW. Detection of silent myocardial ischemia in DM. Am J Cardiol 1991; 67: 1073

[12]. Quek DK. Association of diabetic autonomic neuropathy and painless myocardial ischemia induced by exercise. Singapore Med J 1992; 32(2): 177-81.

[13]. Gupta SB, Pandit RB. Silent myocardial ischemia and cardiac autonomic neuropathy I diabetes. Ind Heart J 1993; 44(4): 227-9.

[14]. Mathura KC, Vaidya B, Gurbacharya DL. Study of serum lipid profile in type 2 diabetic patients attending KMCTH. Nepal Med Coll J 2005; 7(2): 97-100.

[15]. Agarwal R, Sharma P, Pal M, Kochar A, Kochar DK. Magnitude of dyslipedemia and its association with micro and macro vascular complications in type 2 diabetes; a hospital based study from Bikaner (Northwest India). Diabetes Res ClinPract 2006; 73(2): 211-4.

[16]. Achari V, Thakur AK. Treadmill testing in asymptomatic type 2 diabetes. JAPI 2002 Jan; 50: 50-53.

[17]. DeLuca AJ, Saulle LN, Aronow WS, Ravipathi G, Weis MB. Association of hemoglobin A1c with prevalence of silent myocardial ischaemia. Am J Cardiol 2005 June 15; 95(12): 1472-.

[18]. Gautam Ravipathi, Wilbert S. Aronow, Chulahn, et al. Association of hemoglobinA1c level with the severity of coronary artery disease in patients with diabetes mellitus. Am J Cardiol 2006 April 1; 97(7): 968- 969. 